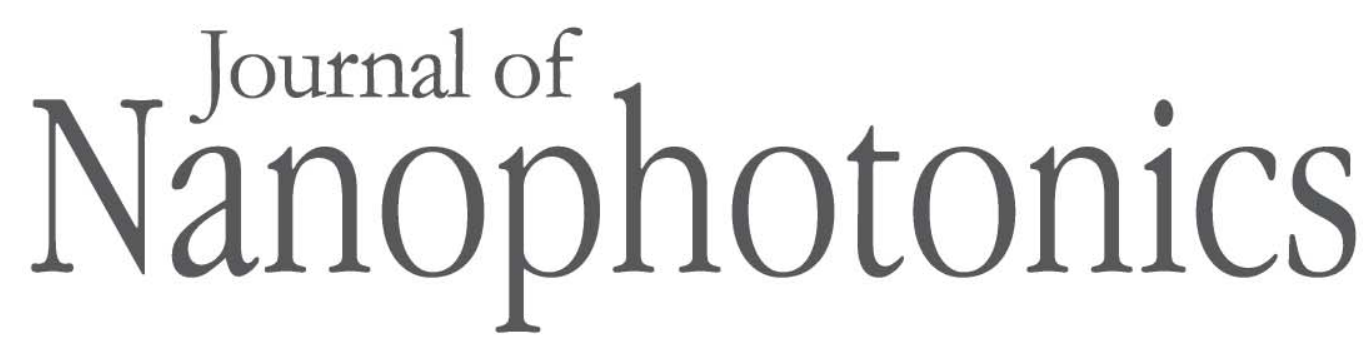

SPIEDigitalLibrary.org/jnp

\title{
Optical properties of nanostructured materials: a review
}

François Flory

Ludovic Escoubas

Gérard Berginc 


\title{
Optical properties of nanostructured materials: a review
}

\author{
François Flory, ${ }^{a, b}$ Ludovic Escoubas, ${ }^{b}$ and Gérard Berginc ${ }^{c}$ \\ ${ }^{a}$ Ecole Centrale Marseille, Technopôle de château Gombert, 38 rue Joliot Curie, \\ 13451 Marseille cedex 13, France \\ francois.flory@ec-marseille.fr \\ ${ }^{\mathrm{b}}$ Aix-Marseille University, Institut Matériaux Microélectronique Nanosciences de Provence \\ IM2NP CNRS UMR 6242, Campus de Saint-Jérôme, Avenue Escadrille Normandie Niemen \\ Service 231, F-13397 Marseille Cedex 20, France \\ ${ }^{c}$ Thalès Optronique S.A., 2 Avenue Gay-Lussac, 78995 Elancourt Cedex, France
}

\begin{abstract}
Depending on the size of the smallest feature, the interaction of light with structured materials can be very different. This fundamental problem is treated by different theories. If first order theories are sufficient to describe the scattering from low roughness surfaces, second order or even higher order theories must be used for high roughness surfaces. Random surface structures can then be designed to distribute the light in different propagation directions. For complex structures such as black silicon, which reflects very little light, the theory needs further development. When the material is periodically structured, we speak about photonic crystals or metamaterials. Different theoretical approaches have been developed and experimental techniques are rapidly progressing. However, some work still remains to understand the full potential of this field. When the material is structured in dimension much smaller than the wavelength, the notion of complex refractive index must be revisited. Plasmon resonance can be excited by a progressing wave on metallic nanoparticles inducing a shaping of the absorption band and of the dispersion of the extinction coefficient. This addresses the problem of the permittivity of such metallic nanoparticles. The coupling between several metallic nanoparticles induces a field enhancement in the surrounding media, which can increase phenomena like scattering, absorption, luminescence, or Raman scattering. For semiconductor nanoparticles, electron confinement also induces a modulated absorption spectra. The refractive index is then modified. The bandgap of the material is changed because of the discretization of the electron energy, which can be controlled by the nanometers size particles. Such quantum dots behave like atoms and become luminescent. The lifetime of the electron in the excited states are much larger than in continuous energy bands. Electrons in coupled quantum dots behave as they do in molecules. Many applications should be forthcoming in the near future in this field of research. $\odot 2011$ Society of Photo-Optical Instrumentation Engineers (SPIE). [DOI: 10.1117/1.3609266]
\end{abstract}

Keywords: nanostructured thin films; surface scattering; photonic crystals; quantum dots; nanoplasmonics; solar cells.

Paper 11069V received Jun. 8, 2011; accepted for publication Jun. 14, 2011; published online Aug. 10, 2011.

\section{Introduction}

From atoms and molecules to crystals and bulk components, optical materials are naturally structured at different scales. Thanks to tremendous progresses in nanotechnologies, the optical materials can also be artificially structured at different scales.

The interaction of materials with optical waves and photons is strongly dependent on the structure, which can then be used to control light field distribution and light propagation. This

$1934-2608 / 2011 / \$ 25.00$ (c) 2011 SPIE 
allows the development of a large range of key components for optical systems and it is now a major field of photonics.

Refraction, interferences, diffraction, scattering, anisotropy, absorption, light emission, and nonlinear effects are all widely used to develop photonic components. Here, we will focus our attention only on a small part of this wide field. Our purpose is to try to have a general look at linear and passive interaction of light with structured transparent materials and to give a personal view on the related phenomena. The applications are numerous and generally belong to what are called information and communication technologies in its wider meaning and green photonics, in particular photovoltaic solar cells.

The electromagnetic theory based on Maxwell equations with a rigorous approach or with more or less simplifying considerations, allows the description of light propagation in complex media when the complex refraction indices of the materials are known. We will see that the notion of refractive index must be revisited in some cases of nanometer structures. With the increase of computer power Maxwell equations can be solved, whatever the structure is, but one has to keep in mind that the sampling of spatial and temporal variables, which is necessarily finite, is still an approximation.

The general aim of photonic components is to control the way light is distributed and propagates, and its polarization and frequency.

We will consider at first surface structures. The roughness of the surface induces light scattering even with a high-quality polishing process. The surface can also be artificially structured with a periodic pattern or a random surface to have antireflecting properties or to control the light direction by diffraction. A brief review of major results in the field is discussed.

Stacking thin films of different materials and different thicknesses is the important field of optical interference coatings. Depending on deposition process and the material, the natural micro/nanostructure of the films can be more or less complex. This has different consequences on their optical properties, which are reviewed. When the films are periodically structured in one- (1D), two- (2D), or three dimensions (3D), one speaks about photonic crystals because light waves behave like the wave function of electrons in crystals. Optical interference coatings can be considered as 1D photonic crystals. Metamaterials also belongs to the photonic crystal field. The name photonic crystal is now used in a wider meaning as it can also concern nonperiodic structures.

Another field of nanophotonics that is strongly developing is the field of nanoplasmonics. It concerns the use of plasmon resonance in small metallic structures of different shapes. A lot of work is performed in this field for different applications like plasmonic circuits, plasmonic solar cells, surface enhanced Raman scattering, or tip enhanced Raman scattering. Another domain that emerged a decade ago and which is another hot topic, concerns the optical properties of quantum structures to make biological tags, efficient light emitting diodes (LEDs), efficient solar cells, or low-consumption flat panel displays. The modeling of the constitutive properties of films including quantum dots cannot be performed by the usual harmonic oscillator model. New approaches taking quantum confinement of electrons into account are discussed.

\section{Surface Structures}

\subsection{Random Surfaces}

Electromagnetic wave scattering from randomly rough surfaces and films has emerged as a discipline of considerable research in areas including surfaces optics and plasmonics. A randomly rough surface is described in terms of statistical parameters. We usually consider that there are two parts that define the surface roughness: its deviation from a plane reference surface and the variation of these heights along the surface. These surface properties are described by statistical distributions as surface height distribution functions and correlation functions that appear in the theory of wave scattering from randomly rough surfaces. In nanoscale roughness region, surfaces with large roughness height and small correlation length will have the steepest 
slopes. Some synthetic surfaces have much larger slopes than natural surfaces. A main tool for studying these surfaces is wave scattering simulation.

Because of its roughness, an optical surface scatters light. In 1956, Giacomo already gave the results of measurements of the angular distribution of light scattered by Fabry-Perot filters showing scattering peaks corresponding to angle of resonances of the filter. ${ }^{1}$ Since then, many works have been performed on scattering theory. For slightly rough surfaces and thin films, Kröger et al. ${ }^{2}$ proposed a first order theory in 1970 . The relation between the angular dependence of scattering and the statistical properties of optical surfaces was later described by Elson et al. in $1976 .{ }^{3}$ Each scattering angle is related to a spatial frequency of the autocorrelation function of surface roughness. In other words, the roughness of the surface is decomposed in a sum of gratings of different steps. A spatial frequency corresponds to a step of a grating and then a scattering direction. The depth of each grating determines the scattering amplitude.

When concerned with multilayers made of homogeneous thin films, the roughness of each layer boundary must be taken into account. The roughness of one boundry is linked to the next with an intercorrelation function. Depending on the spatial frequency, the deposition technique, and on the material considered, this intercorrelation function can be close to one. Both a first order theory and measurement of scattering from multilayer thin films have been given by Bousquet et al. in $1981 .{ }^{4}$ In this theory, a slightly rough surface is replaced by a plane surface holding a surface distribution of currents. These secondary sources send light in different directions for which the multilayer has different optical properties. The scattering diagram results from both the amplitude of these secondary sources and the transmission of the stack.

Different theoretical and numerical approaches have been developed to model the scattering of an electromagnetic field by random rough surfaces. The small-perturbation method ${ }^{5}$ (SPM) is used for surfaces with small roughness. An overview of the method for one-dimensionally rough surfaces is given by O'Donnel in chapter 5 of Ref. 6 . The Kirchhoff approximation method ${ }^{7}$ is used for long correlation length surfaces, the method is described by Voronovich in Ref. 8 and in chapter 2 of Ref. 7. We can also cite the full-wave method analysis developed by Bahar, ${ }^{9}$ the surface-field phase-perturbation technique developed by Ishimaru and Maradudin, ${ }^{10,11}$ and the quasi-slope approximation developed by Tatarskii. ${ }^{12}$ Research on numerically exact methods for randomly rough surfaces has increased over the past quarter century. Modeling rough surface scattering by exact methods often involves the choice of a one-dimension surface model due to the reduced computational complexity for these types of surfaces. But one-dimensional models are inadequate for a set of scattering effects such as cross-polarized scattering and scattering outside the plane of incidence. In the last decade, results have also been obtained by rigorous computational approaches for the scattering of polarized light from 2D randomly rough surfaces (see chapter 7 in Ref. 7 for an overview). For a randomly rough surface (i.e., stochastic problems), one can compute averages of scattered fields obtained by an ensemble of surface realizations and direct examinations of convergence are often appropriate. Problems with very large surface and Monte Carlo averaging require supercomputing resources to obtain results within a reasonable amount of time. Comparison with results obtained by analytical predictions of randomly rough surface scattering can give some insight about the accuracy of numerical computation, for example, in the case of small magnitudes of scattered intensity. We can notice that in studies of scattering from nanoscale rough surfaces, the goal is to understand the physical scattering process, subtle phenomenon whose magnitude and angle range are not very large, therefore, new analytical techniques must be developed rather than extensive use of numerical exact methods.

In the mid-1980s, a new popular method, called the small-slope approximation (SSA), ${ }^{8,13-16}$ was proposed by Voronovich. The SSA is an appropriate candidate to bridge the gap between the Kirchhoff approximation and the small-perturbation method. It is valid for arbitrary roughness, provided that the slopes of the surface are smaller than the angles of incidence and scattering. This method has been extended up to the third-order to 3D vector cases for Gaussian and non-Gaussian randomly rough surfaces. The coherent and incoherent component formulations of the electromagnetic intensity for cross- and co-polarization have been developed in bistatic 
and monostatic cases. ${ }^{15}$ The SSA method has also been used to study different rough structures like a 3D slab or a film, considering the effects of higher orders in a perturbative expansion. The small slope approximation has been extended to the fourth-order terms of the perturbative development, and the expression of the cross-sections for the different polarization states can be found in Ref. 16. New formulations of the SSA are described in Ref. 16. In the development of the SSA series, the third-order SPM kernel has been taken into account. An expression of this kernel is developed in chapter 6 of Ref. 7 for 2D surfaces and a 3D slab, the development up to third order of the SPM is mandatory to analyze the phenomenon of backscattering enhancement, a well-defined peak in the retro-reflection direction of incidence for the case of nanoscale surface roughness. The Voronovich SSA was generalized to a layer bounded with two randomly rough surfaces. New terms in the SSA were introduced to consider the coupling between the two rough surfaces. Complete expressions of the scattering matrices and the expression of the needed crosssection for the different polarization states have been developed. ${ }^{16}$ With this new formulation of the SSA, the backscattering enhancement for a slightly rough layer can be calculated and new analytical theories have been developed to understand the backscattering enhancement effects. ${ }^{17}$

The last problem is described by random volume and surface scattering. Useful phenomena in the optical range can be produced by random media with randomly rough surfaces. Designing these disordered slabs with rough surfaces can produce new optical components, which can transmit or scatter optical field with specified angular, spatial, or spectral properties. Understanding how light interacts with disordered matter is a fundamental issue in optoelectronics and photonics and has huge consequences in communications, imaging, and sensing. New formulations have been developed for a three-dimensional disordered medium with randomly rough interfaces. ${ }^{18}$ This structure describes a device based on metallic nanoparticles embedded in insulators or dielectric media. A theory of transport based on the Bethe-Salpeter equation is presented in Ref. 18. For a three-dimensional system composed of a random medium bounded by two randomly rough surfaces, the Bethe-Salpeter equation is constructed in order that the medium and the boundaries are treated on the same footing. With this unified Bethe-Salpeter, a general expression is obtained, whatever the choice of the scattering operators used at the boundaries.

This type of simulation computation can give some experimental conditions and specifications to realize highly integrated optical devices that use metallic or metallo-dielectric nanoscale structures. For 3D slabs with nanoscale roughness and nanoparticles, the enhanced backscattering is produced by different mechanisms, the wave scattering by the same boundary, the wave coupling by the two boundaries, or the wave scattering by the random volume. All of these models and simulations can demonstrate that plasmon-polariton resonances are responsible for remarkable optical phenomena.

\subsection{Controlled Random Surfaces}

The control of surface roughness with tuned statistical properties is a mean to choose the scattered light distribution. ${ }^{19}$ The method based on photofabrication using the speckle of a diffused laser beam has been proposed by Gray. ${ }^{20}$ It allows tuning the statistical properties of the generated surface by modifying parameters of the exposure. Several other results based on this method have been derived after Refs. 21-23, in particular for extending depth of focus or light uniformization. ${ }^{24}$ More recently, a method based on a spatial light modulator to control the laser beam shape appeared to be flexible. ${ }^{25}$

Controlling the stochastic parameters of randomly rough surfaces can be a method to "design" the scattering of an electromagnetic wave. For example, the problem is how to design a 2D randomly rough surface that scatters, in a specified manner, an optical wave incident on it and the surface realized is not a deterministic one. We notice that this kind of process is an inverse one; we must determine the structure of the surface considering the pattern of the scattered wave and the mean intensity of the scattered field, which is regarded as a spatial average of 
the scattered intensity over an area containing many realizations of the surface roughness. A subtle question arises when we compare the definition of wave scattering from a randomly rough surface. In the case of a theoretical computation, the scattered field is averaged over the ensemble of realizations of the surface profile function. The measurement is considered on a single realization of the surface profile function. The assumption that the spatial average is equivalent to the ensemble average, can only be proven if the generated surfaces are stationary and it is not always the case. The validity of this assumption is common to all experimental work.

\subsection{Black Silicon}

Black silicon has been studied for more than 10 years. Black silicon texture has been produced by techniques including laser-chemical, ${ }^{26}$ electrochemical, ${ }^{27}$ and reactive-ion etching. ${ }^{28}$ Whatever the production technique, a black silicon surface generally exhibits quasiperiodical conical structures of height and a period of a few microns (Fig. 1). In some cases, irregular nanometer structures on the top of the cones make the surface more complex. Such a surface has been obtained by Mazur et al. ${ }^{29}$ by sending femtosecond UV laser pulses in a $\mathrm{SF}_{6}$ gas on a silicon surface. Sulphur is embedded into the silicon surface. Black silicon presents remarkable wide band antireflection properties and makes the silicon highly absorbing both in the visible and in the near infrared, below silicon band gap. ${ }^{30}$

Wet etching of silicon using a chloroauric acid bath is also an inexpensive way to make black silicon.

Reactive ion etching has also been used to replicate a photoresist pattern on a silicon substrate. Troconical pyramidal shapes made by photolithography with one or two periods also gives a very wide band antireflection silicon surfaces. ${ }^{31,32}$ Other techniques to make black silicon do not need photolithography. The masks are made of silica or polystyrene ${ }^{33-35}$ particles directly deposited on silicon.

If the remarkable properties of black silicon are not yet well explained, it seems that both the structure and a possible high doping of the material may play a role. A question that is still opened concerns the smallest structures, as nanometer structures may induce quantum confinement effects.

A major issue arises with black silicon: can black silicon be the inexpensive way toward visible-short IR wavelength detectors? Besides $\mathrm{HgCdTe}$ and InGaAs, black silicon is a new way to capture visible, near infrared, and small wave infrared light.

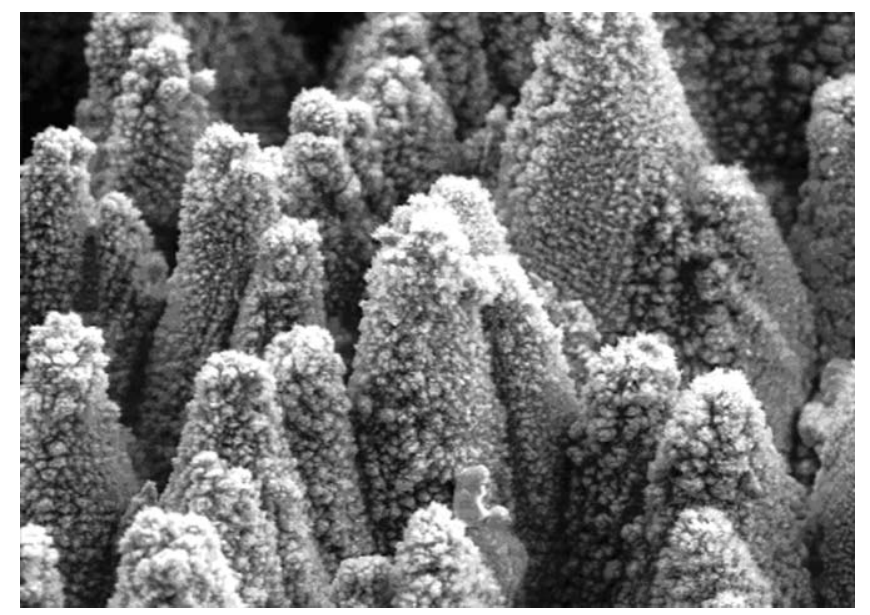

Fig. 1 Low resolution SEM of the black silicon surface (Ref. 29). 


\section{Thin Film Structures and Optical Coatings}

The use of interferences in multilayer coatings has been developed for many years to reduce the reflection of an optical surface, to make wavelength filters of different types like mirrors, band pass, high pass, low pass, etc. Optical coatings are now widely used in optical systems and very often they are key elements for the implementation of very important applications. Between others, low-loss mirrors are in production for gyrolasers used in automatic navigation systems. Very narrow-band pass filters are key components for dense wavelength division multiplexing optical communications or for astronomy. A very rich literature is available on this subject. ${ }^{36}$

Depending on the deposition technique used, the micro/nanostructure of thin films can be more or less complex. It is well known that low energy evaporation condensation techniques based on e-beam gun or on heated crucible give columnar thin films for most of the materials. ${ }^{37}$ The typical dimension of a column is in the order of $20 \mathrm{~nm}$ and in some cases substructures of smaller dimension can be detected. ${ }^{38}$ The refractive index is generally smaller than the refractive index of the bulk material. This is attributed to the lacunar structure of these films. In particular columnar thin films have a packing density that can be much smaller than one. The packing density is given by the ratio of the volume occupied by bulk material over the total volume of the layer.

In air, even for low moisture, water is adsorbed in the pore of the material, leading to an increase of the refractive index and then to a red shift of the optical properties of interference filters. ${ }^{39}$ Under such conditions, thin film materials are made of a mixture of bulk material and vacuum or water. The problem of the refractive index of these mixed materials induced a lot of work some years ago and different models ${ }^{40-42}$ taking into account the mixing have been proposed without giving a very satisfactory solution. In particular, the change induced by water adsorption cannot be fully explained. Porous films can be seen as a mixture of two materials and, following the homogenization theory, the permittivity $\varepsilon$, i.e., the square of the refractive index, of the mixture is the weighted meaning value of the $\varepsilon_{1}$ and $\varepsilon_{2}$ of the two materials. The weights are the proportions of the volume occupied by each material.

This homogenization theory was first proposed by Rytov. ${ }^{43}$ In particular, he has shown that a periodic stack of films, very thin relative to the wavelength, with alternating high and low refractive indices behaves like a single-axis homogeneous material with the optical axis perpendicular to the surface.

Columnar thin films also exhibit a form birefringence. When the deposition is performed with an oblique incidence of vapor (angle $\alpha$ in front of the normal to the surface), the columns are inclined (angle of $\beta$ ) (Fig. 2). The relation between $\alpha$ and $\beta$ is approximately given by $\tan \alpha$ $=2 \cdot \tan \beta$ for $\alpha<45^{\circ}$. The anisotropy increases with an increasing angle ${ }^{44}$ and the material has a biaxial behavior. ${ }^{45,46}$ When the angle $\alpha$ slowly changes during deposition, the material can have a zig-zag, an S, or a helicoidal nanostructure with a nematic or chiral behavior. ${ }^{47-49}$ Such "sculptured thin films" can be classified as nanoengineered metamaterials.

The optical properties of stacks made of anisotropic layers can be calculated by the use of a $4 \times 4$ matrix formalism. ${ }^{50,51}$ Polarizing filters can be made with these materials like halfwave plates $^{52}$ or polarization rotator in reflection. ${ }^{53}$

With ion assisted deposition or ion beam sputtering, the deposition processes are more energetic and the materials do not exhibit columns any longer. ${ }^{38}$ As they are denser, they are also insensitive to moisture.

Light can also propagate in the thickness of transparent multilayers, being trapped by total internal reflection. ${ }^{54}$ The modes are then guided and the propagation constants can take only a finite number of values. A particular electric field distribution corresponds to each guided mode with the guided energy more important in one layer, several layers, or in the whole stack. A guided mode can be seen as a resonance of the optical field in the direction perpendicular to the layer boundaries. The guided mode propagation conditions correspond to constructive interferences in the direction parallel to the layer boundaries. They also correspond to poles of the reflection coefficient. The guided modes can be excited with a prism and this has been 
widely used as characterization techniques as well for refractive index measurements ${ }^{55,56}$ as for loss measurement. ${ }^{54}$ The m-line technique is now developed for gas sensing. ${ }^{57}$

\section{Photonic Crystals}

For optical coatings, the light is reflected or transmitted through the sample. On the contrary, diffraction structures are generally used to distribute the light in a controlled way in different directions of the space. The association of diffractive and interference structures in 3D components allows control, as well the directions, of propagation as the spectral distribution of the optical waves in the space. In particular, the local field and the propagation in the structure can be adjusted with the photon lifetime. The typical dimensions of the structure are in the order of a part of the wavelength. These periodically structured materials are also named photonic crystals because light waves similarly behave to the wave function of electrons in crystals. ${ }^{58,59}$ Another way to describe the light behavior in such structures is to use the density of optical modes as Purcell defined the density of modes for electrons in solid. ${ }^{60}$

Photonic crystals can also be considered as metamaterials with optical properties impossible to achieve with nonstructured materials. As an example, the structure can behave like a material having a refractive index of zero or even negative. Many laboratories and companies pay a lot of attention to the study of metamaterials as important applications may come soon with cloaking properties and stealthiness or perfectly stigmatic optics. It is interesting to consider multilayer mirrors not only as an interference system but also as a diffractive component. A mirror is a periodic stack made of alternating high and low refractive indices, each layer having an optical thickness of a quarter wave for the centering wavelength. It can be considered as a Bragg grating. This concept can be extended to Bragg grating in waveguides. The main difference is that each half period must be quarter wave but for an optical thickness defined with the effective index of the guided mode. This principle can be used to design waveguide filters and well known optical interference coating design methods can be helpful. ${ }^{61}$

As a mirror does not transmit a part of the spectrum, it has a forbidden frequency band for photons and it is a 1D photonic crystal. Thin film Fabry-Perot filters can also be seen as photonic bandgap structures with a defect.

\section{Optical Properties of Materials Including Quantum Structures}

Generally, models used to study the optical properties of nanostructures are based on the electromagnetic theory, but when the dimension of a semiconductor nanocrystal is smaller than the de Broglie wavelength, quantum phenomena must be considered. The de Broglie wavelength can be seen as the coherence length of the electron wavefunction.

Because of their interesting electronic and luminescent properties, plenty of research concerning quantum structures is performed in different laboratories. ${ }^{62} \mathrm{~A}$ structure limited to a few $\mathrm{nm}$ in the three dimensions of space is called quantum dot (QD). QDs have an atom-like behavior, as the energies of the electron or hole can only take discrete sets of values. Their absorption and luminescent spectra depend both on the properties of the semiconductor used and on the size of the QDs. They can be very efficient light emitters with photoluminescence quantum efficiency as high as $80 \%$. Their emission can be continuously tuned through a large spectrum by changing their size. As an example, CdSe QDs emit anywhere from 470 to $630 \mathrm{~nm}$ by varying their size from 3 to $6.5 \mathrm{~nm}$.

Apart from applications already implemented such as multiple quantum wells ${ }^{63}$ for lasers and detectors, ${ }^{64}$ other important applications are forthcoming with the study of quantum structures.

Among others, QDs can be used to make biological tags. ${ }^{65}$ More recently, they have been used to develop high efficiency white LEDs. ${ }^{66}$ They are also under study to make more efficient OLEDs (QLED). ${ }^{67}$

Quantum structures are also studied to increase the photovoltaic solar cell efficiency and to go beyond the Shockley-Queisser limit of $32 \%$ for Si based solar cells. ${ }^{68}$ 


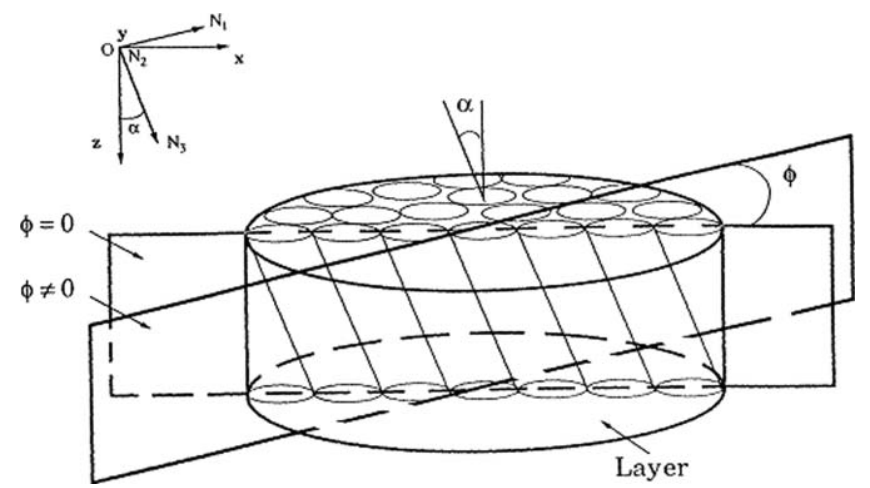

Fig. 2 Model of anisotropy for columnar thin films.

There are two ways for enhancing the conversion efficiency. The photovoltage or the photocurrent can be increased. To increase the photovoltage, the hot carriers must be extracted from the photoconverter before they cool. The energetic hot carriers must produce two or more electron-hole pairs through impact ionization to increase the photocurrent.

In principle, QDs can be used in these two ways, which exclude one to the other in different QD solar cell configurations. ${ }^{69}$ Another way to collect UV photons is to change their frequency to convert them into visible photons. QDs can also be used to make quantum dot-sensitized nanocrystalline $\mathrm{TiO}_{2}$ solar cells. They can also be organized in a thin layer with an absorption band complementary to the absorption band of another material composing the solar cell. ${ }^{70}$

In most photonics components, quantum dots are embedded in thin films. So, to model the optical properties of such components, the refractive index $n$ of thin films including quantum dots (TFIQD) must be known. The classical model used to describe $n$ cannot apply to quantum structures and the quantization of the electron energy must be taken into account.

\subsection{Frequency Conversion}

If the radiative recombination rates of electrons generated by energetic photons are faster than cooling rates, frequency conversion can occur and can increase cell efficiency.

As an example, when considering wide band gap semiconductor nanocrystals like $\mathrm{ZnO}$, the UV can be absorbed and changed into longer wavelengths. Green and UV are emitted. The luminescence in the green is not completely explained but it can be due to oxygen vacancies. ${ }^{71}$ The luminescence in the UV is coming from quantum confinement with an energy level close to the edge of the bandgap. The UV shift of the bandgap is well explained by calculation (Fig. 3). ${ }^{72}$

In this case of luminescence, the charges generated by the UV photons must radiatively relax before being transferred to the surrounding media, being recombined at the nanocrystal surfaces, or being coupled to phonon nonradiative decays.

\subsection{Charge Carrier Generation}

Depending of their size, CdSe nanoparticles produce electrons and holes from different photon energies of the visible spectrum. The charges can be transferred to the surrounding medium if resonant transfers are possible. These particles can replace dye molecules in $\mathrm{TiO}_{2}$ sensitized dye. ${ }^{73}$ It is interesting to notice that, thanks to the relative band offset, CdSe quantum dots have a resonant electron energy level with the conduction band of $\mathrm{TiO}_{2}$. "Rainbow" solar cells using $\mathrm{CdSe}$ of different-sized quantum dots assembled in an orderly fashion are under study. ${ }^{74}$ 
Flory, Escoubas, and Berginc: Optical properties of nanostructured materials: a review

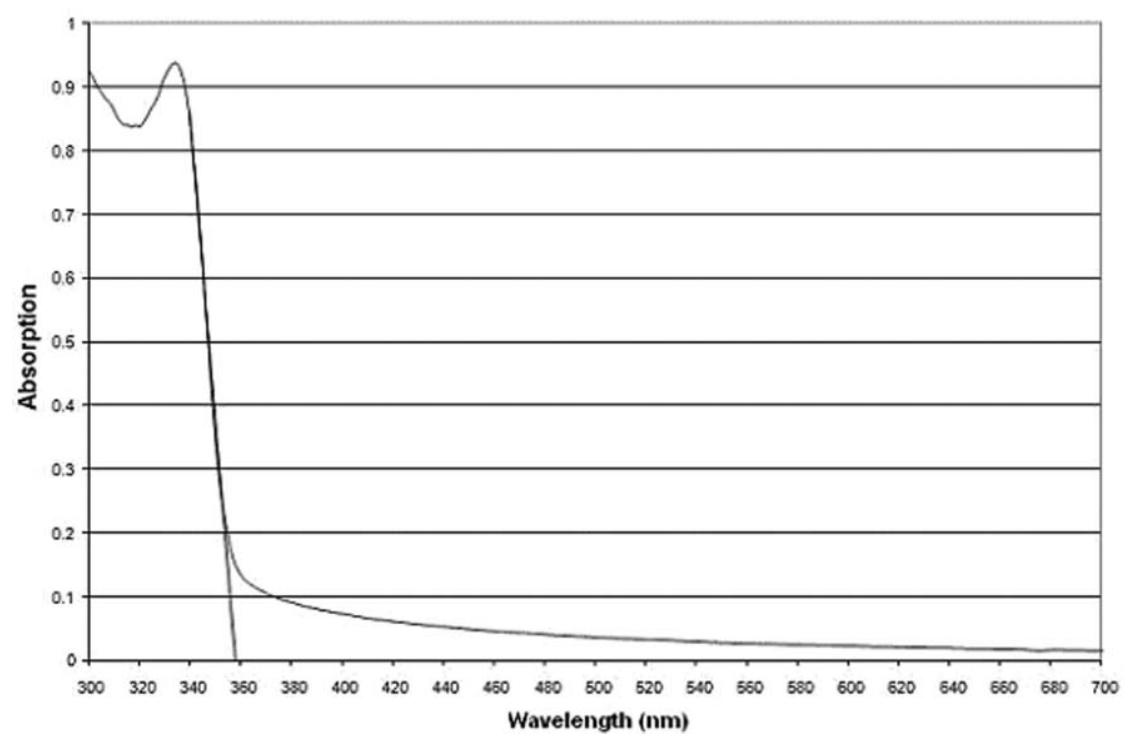

Fig. 3 Absorption spectrum of $\mathrm{ZnO}$ nanocrystals in a solution of $\mathrm{MeOH}$.

Hybrid organic/inorganic cells can also be made with P3HT and CdSe nanorods or P3HT and $\mathrm{PbS}$ nanoparticles. With size-quantized $\mathrm{PbS}$ nanoparticles, 3\% incident photon to current efficiencies (IPCE) under 550-nm monochromatic irradiation is obtained. ${ }^{75}$ A $6.9 \%$ IPCE under 0.1 milliwatt per square illumination at $515 \mathrm{~nm}$ is obtained with 7 -nanometer by 60 -nanometer CdSe nanorods. ${ }^{76}$

\subsection{Multiple Exciton Generation}

When photoexcited carriers elastically collide with one another, no energy is lost. On the contrary, collisions with the atoms of the cell material are inelastic. They induce an energy loss with phonon induced decays. In principle, if atomic collisions can be avoided during the time it takes a photogenerated carrier to cross the cell, such a thermalization loss can be avoided. Carriers either have to go through the cell very quickly or cooling rates have to be slowed in some way. If, instead of giving up their excess energy as heat, the high energy electron-hole pairs are used to create additional pairs, higher efficiency would be possible (Fig. 4). This can be achieved by using QDs because of the longer lifetime of the discrete excited states. The creation of more than one pair by high energy photons has attributed to impact ionization by the photoexcited carriers. In such a case, many electron-hole pairs could be generated by each incident photon (Fig. 5). ${ }^{77}$

In principle, MEG can give up to $75 \%$ efficient conversion. To the best of our knowledge, only the Auger principle has been demonstrated, but there may be different competing processes. Charge cooling because of phonon coupling is, of course, one of them but also, if the absorption occurs into the wrong state, there will be no impact ionization. Auger recombination after decay can also happen.

\subsection{Optical Properties of Thin Films Including Quantum Dots}

The value of the complex refractive index or permittivity is needed to calculate the optical properties of photonic structures. The bulk value of the refractive index is not fitted when the materials are structured in small dimensions and the classical model ${ }^{78-80}$ cannot be used. 
Flory, Escoubas, and Berginc: Optical properties of nanostructured materials: a review

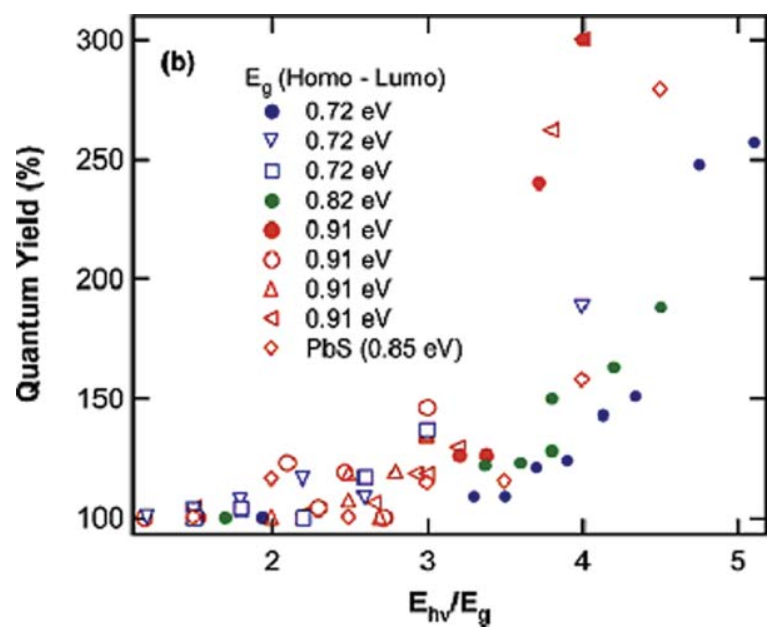

Fig. 4 Quantum yield versus the relative energy of photons in front of the material bandgap showing multiple exciton generation with $\mathrm{PbSe}$ nanoparticles.

A model for quantum wells can be used as a first theoretical model. Many works have already been performed on single and periodic quantum wells of square and nonsquare profiles. ${ }^{81,82}$ The model presented in Ref. 72 based on the approximation of the effective mass, can be applied to single wells, coupled periodic, and nonperiodic multiple wells and to stacks of wells of any depth and any width. The main advantages of this model are its easy implementation and its small computation time.

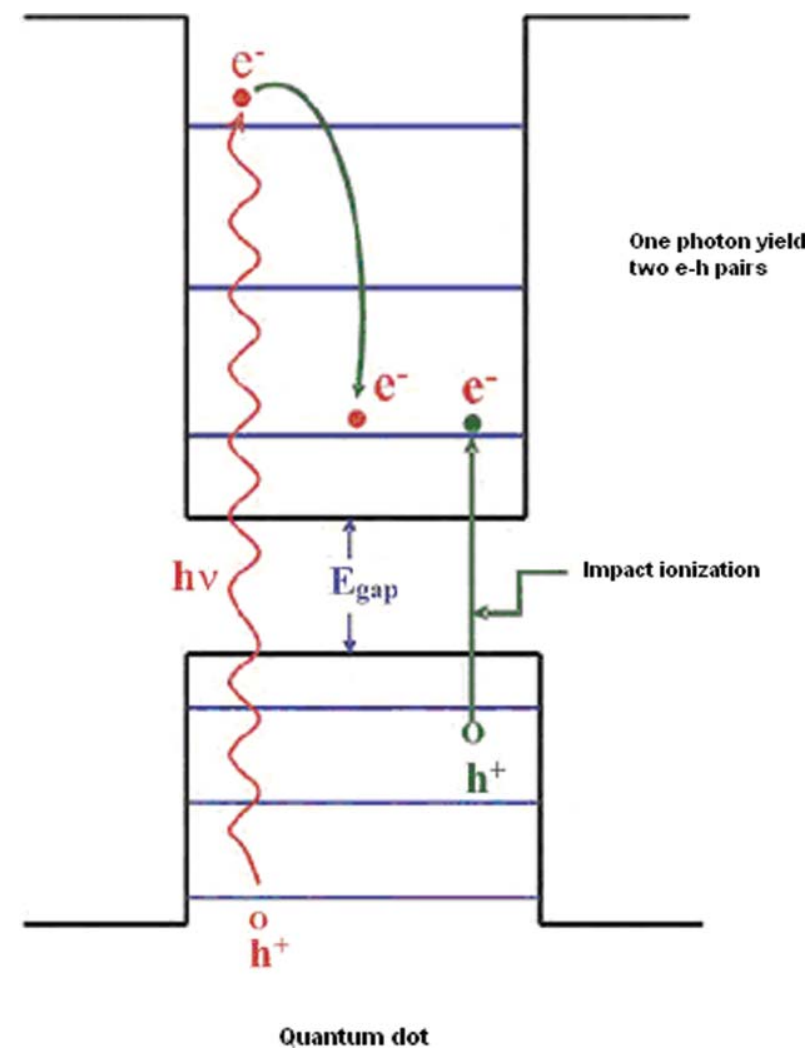

Fig. 5 Sketch of a multiple exciton generation process. 
Flory, Escoubas, and Berginc: Optical properties of nanostructured materials: a review

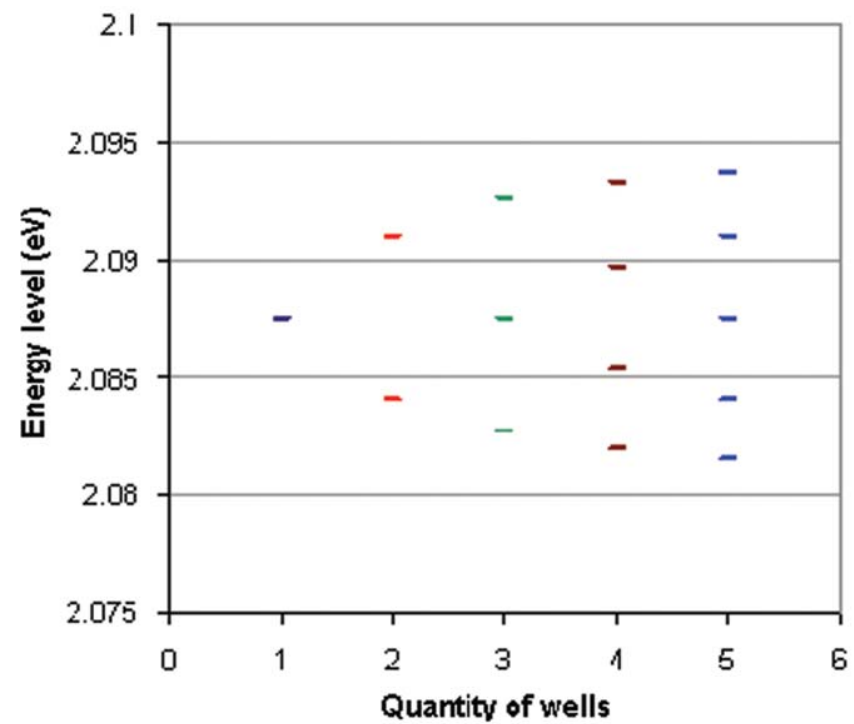

Fig. 6 Splitting of the highest energy level of electrons in coupled quantum wells, when considering from left to right $1,2,3,4$, and 5 identical coupled wells. Each well has a depth of $2.9 \mathrm{eV}$, a width of $1 \mathrm{~nm}$ and the distance between the wells is also $1 \mathrm{~nm}$.

In a multiple well, a recurrent relation can be found for the function $Q=\frac{1}{m} \frac{d \psi}{d x} / \psi$, where $\psi$ is the electron wave function and $x$ is the direction perpendicular to the well boundaries

$$
Q_{j}=k_{j} \frac{Q_{j-1}-k_{j} \tan \left(k_{j} a_{j}\right)}{Q_{j-1} \tan \left(k_{j} a_{j}\right)+k_{j}},
$$

with $j$ referring to a well boundary in the structure. The width of the $j$ th layer is $a_{j}$ and $k_{j}=\chi_{j}$ for $E>V_{j}$ or $-i \gamma_{j}$ for $E<V_{j}$. The function $Q$ is continuous, crossing a potential barrier. So, the value of $Q$ on one limit of a structure can be obtained as a function of $Q$ on its other limit.

If the electron is confined in the structure, $\psi$ is evanescent in the surrounding media. The equation describing the possible discrete energy levels is then derived from these considerations.

The wavefunction and the square of its modulus giving the electron density probability distribution, can then be calculated in the whole structure

$$
\Psi_{j}(x)=C_{j}^{+}\left[e^{i k_{j} x}+\frac{i k_{j}-Q_{j-1}}{Q_{j-1}+i k_{j}} e^{i k_{j}\left(2 x_{j-1}-x\right)}\right],
$$

where $C_{j}^{+}$is a constant that can be normalized. $k_{j}=\chi_{j}$ for $E>V_{j}$ or $-i \gamma_{j}$ for $E<V_{j}$ with $\chi_{j}^{2}=2 m / \hbar^{2}\left(E-V_{j}\right)$ and $\rho_{j}^{2}=2 m / \hbar^{2}\left(V_{j}-E\right)$. For each possible value of the energy $\int|\psi|^{2} d x=1$.

In identical coupled wells, the energy levels of the single well are split (Figs. 6 and 7) and when there are a large number of coupled wells, they become energy bands. In nonperiodic structures, the electrons can be localized or delocalized like electrons in molecules (Fig. 8). It is worth noticing that the properties of the charges can then be engineered, to some extent, by adapting the size of the QDs and their coupling distance.

A lot of new applications are expected to come with such artificial molecules.

As for electrons, the energy levels of the holes can be calculated in quantum wells. The optical properties of quantum wells can then be deduced from the electron and hole energy distribution. An example of a model is proposed in Ref. 83 for the complex refractive index dispersion with a wavelength of interdiffused quantum wells. In the case of a film composed of quantum dot semiconductor embedded in a homogeneous matrix, the refractive index can be 
Flory, Escoubas, and Berginc: Optical properties of nanostructured materials: a review

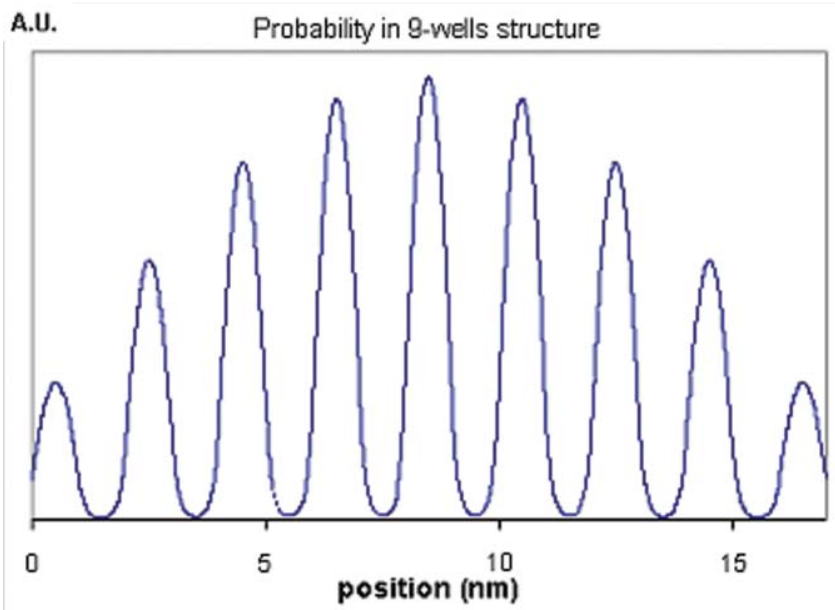

Fig. 7 Electron density of probability for the lowest energy level in a structure composed of nine identical coupled wells.

calculated from the refractive index of the quantum dots and the refractive index of the matrix by using the homogenization theory.

Knowing the energy levels of electrons and holes in a quantum structure ${ }^{84}$ and taking into account the band broadening phenomena induced by size dispersion and coupling effects, the absorption dispersion with wavelength can be approximately calculated. But the amplitude of the absorption in QDs cannot be directly calculated as it depends on their absorption cross section, which has not yet been modeled. The absorption amplitude can then be only obtained by measurement. By measuring $R(\lambda)$ and $T(\lambda)$ of a thin film including quantum dots, the extinction coefficient dispersion is deduced from the absorption $A(\lambda)=1-R(\lambda)-T(\lambda)$

$$
k(\lambda)=-\frac{\lambda}{4 \pi d} \log (A(\lambda))
$$

with $d$ the thickness of the film. By using Kramers-Krönig relations, the dispersion of the real part of the refractive index of the TFIQD can be obtained. As to have quantum confinement,
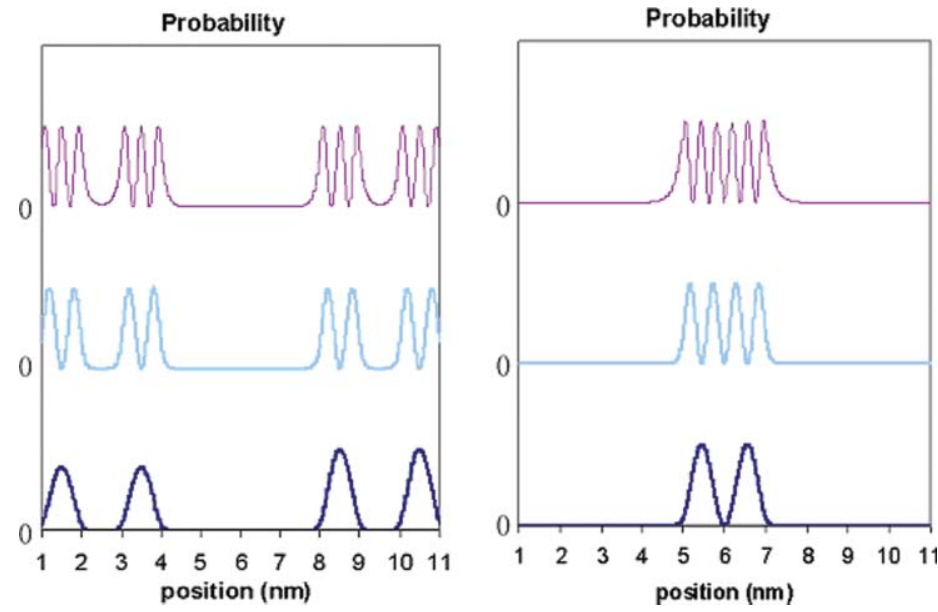

Fig. 8 Electron density of probability in a nonperiodic structure composed of five coupled wells of depth $2.9 \mathrm{eV}$, of widths $1,1,2,1$, and $1 \mathrm{~nm}$, respectively. The distance between each well is $1 \mathrm{~nm}$. 
the bandgap of the QDs must be smaller than that of the surrounding material, the absorption is mainly due to the QDs for the wavelengths just shorter than the QDs bandgap. In this spectral region, the measured absorption dispersion with wavelength should correspond to the calculation.

So, research on quantum structures for photovoltaic cells is very active. In the near future, many progresses are expected to develop high-efficient and low-cost solar cells. The control of the material structures at a nanometer level is mandatory to fully benefit from the attractive properties of QDs of different sizes and made of different materials. Coupled QDs could also lead to new useful structures for many different applications.

\section{Nanoplasmonics}

The free electrons at a planar surface between a metal and a dielectric material can exhibit a collective oscillation of free electrons at a resonant frequency. The corresponding surfaceplasmon-polariton (SPP) electromagnetic wave of TM polarization propagates along the surface. This surface wave can only be excited with an evanescent wave. A totally reflecting prism can be used for that purpose. ${ }^{85}$

When considering metallic nanoparticles of dimension of a few tens of nanometer, the SSP can be excited on the nanoparticle surfaces. ${ }^{86}$ The two key interest areas are 1 . the ability to channel light into sub-wavelength zones and 2. the local optical electric field is strongly enhanced on the metal surface thanks to plasmon resonance. This can be used to increase the absorption in the surrounding material or to enhance fluorescence and Raman scattering.

As the propagation length of a SPP is generally longer than the circumference of the metallic nanoparticle, the electric field amplitude is modulated on the surface. If several nanoparticles are close enough to be coupled, the optical electric field is also increased in the medium between the nanoparticles. The resonance and its width depend, of course, on the metal used but also on the size, the shape of the nanoparticle, and the dielectric properties of the surrounding medium. It can take place in the visible or in the infrared. The SPPs has a TM polarization when the dielectric media is isotropic but multiple modes of SPP-wave propagation with different polarization states can be simultaneously supported by a metal/structured thin film interface. ${ }^{87}$

The plasmonics features have been extensively studied by the international scientific community and are considered as extremely promising..$^{88}$ Optical nanoantennas based on metallic nanostructures will boost the efficiency of light-matter interactions. ${ }^{89}$ This electromagnetic enhancement plays a key role in surface enhanced techniques: surface enhanced Raman spectroscopy, surface enhanced fluorescence, surface enhanced infrared absorption, etc. Using these techniques, single molecule detection can now be achieved. ${ }^{90}$ The correlation between the observed electromagnetic enhancement and the plasmonic properties of these nanoantennas is heavily studied.

Highly efficient plasmonic nanostructures are also required for further development of new optical near-field techniques such as tip enhanced Raman spectroscopy.$^{91}$

Nanoplasmonic is also under study to make plasmonic optical circuits, ${ }^{92}$ highly efficient light emitting diodes, ${ }^{93}$ and extremely sensitive chemical and biochemical sensors. ${ }^{94}$

Plasmonics has a great interest in the photovoltaic domain in order to improve the photonic absorption, thus, the solar cell efficiency. ${ }^{95}$ For this application, the aim of plasmonics is to excite localized plasmons by using metallic nanoparticles to trap or confine light inside the photoactive material, or to obtain beneficial resonant internal light scattering on these metallic particles. ${ }^{96-98}$ This excitation depends on several parameters such as the metal nature, the surface density of the particles, their diameter, the incident angle of light, the wavelength, the polarization, etc. Light absorption in the active material can then be increased, in particular in the spectral ranges where the photovoltaic material only weakly absorbs the light. As an example, one of the best power efficiencies for an organic solar cell [6.5\%, Ref. 99] is based on an active donor:acceptor bulk heterojunction, the P3HT:PCBM. Its low cutting wavelength (close to $650 \mathrm{~nm}$, i.e., a bandgap around $2.1 \mathrm{eV}$ ) greatly limits the efficiency. Enhancing light absorption around this spectral 


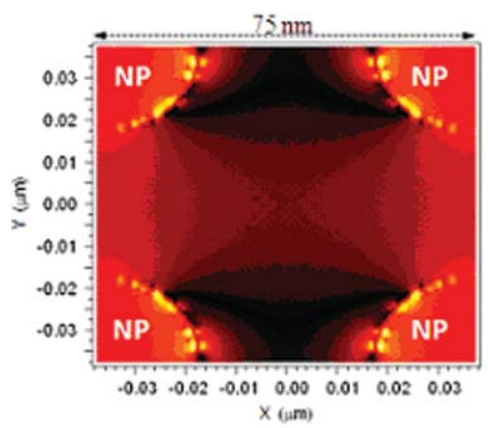

(a)
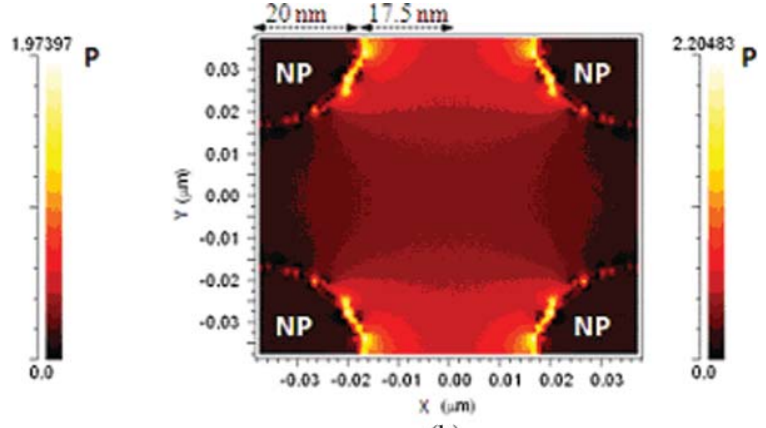

(b)

Fig. 9 Electromagnetic power density distribution in the plane $\{x, y\}$ for an array of four silver nanoparticles (NPs) embedded in a dielectric material. Incident wavelength: (a) 450 and (b) $600 \mathrm{~nm}$.

range where solar intensity is maximum is the current challenge. As a consequence, a precise and highly efficient characterization of optical nanoantennas used in such devices is essential and can be seen as a real challenge. Unfortunately, it remains a "largely unexplored terrain." 88

Today, many theoretical calculations [finite-difference time-domain (FDTD), for instance] have clearly demonstrated the nature and the spatial localization of the electromagnetic field in the vicinity of metallic nanoparticles. As an example, the electric field distribution calculated with the FDTD method in a poly [2-méthoxy-5-(2-éthyl-hexyloxy)-1, 4-phénylène-vinylène]: 6, 6-phenyl C61-butyric acid methyl ester (MEH-PPV:PCBM) bulk heterojunction including silver nanospheres is shown in Fig. 9. A comparison between the absorption with and without nanoparticles of such a heterojunction is given in Fig. 10. ${ }^{100,101}$

Nevertheless, it remains extremely difficult to have an experimental nanometer localization of the enhanced electromagnetic field. In classical surface enhanced spectroscopy, the final optical signal is measured in far field and the precise localization of hot spots remains impossible due to the poor resolution. As a consequence, it appears extremely difficult to validate the nature of the enhanced electromagnetic field obtained with theoretical calculations by experimental

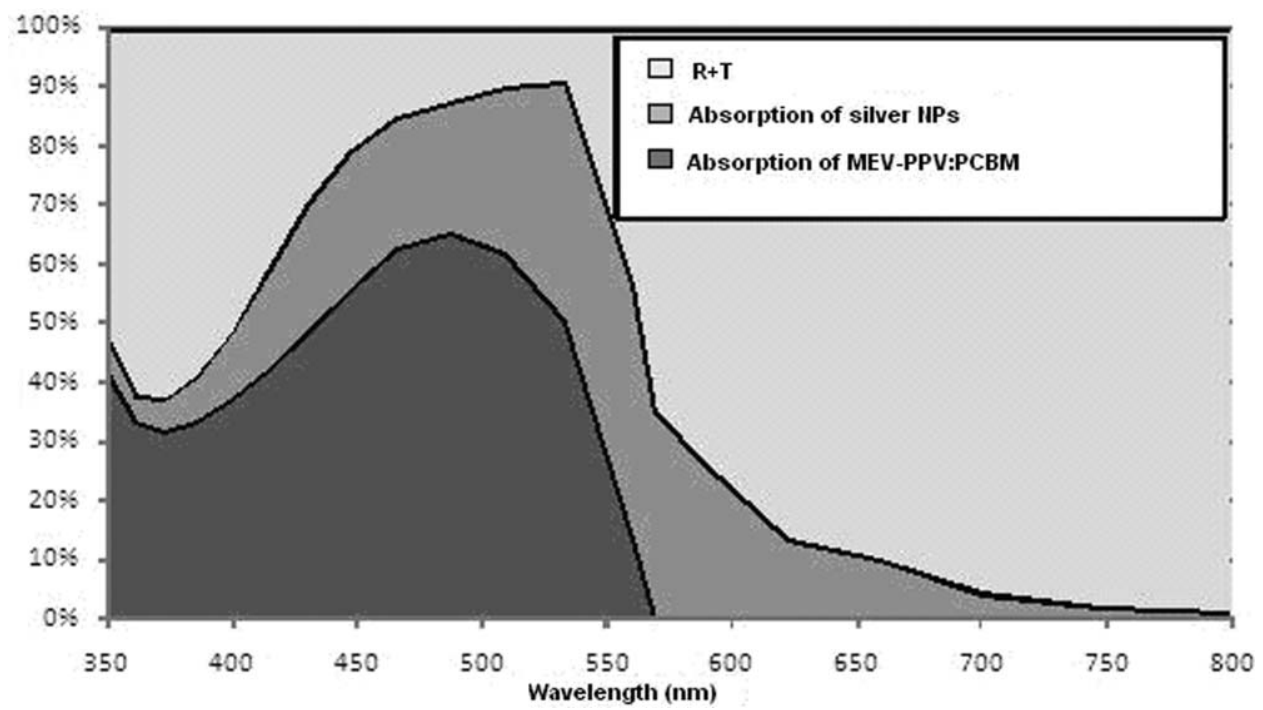

Fig. 10 Intrinsic absorption of MEH-PPV:PCBM (light gray), silver NPs (black), and absorption losses (reflection and transmission) (dark gray) for a structure with NP diameters of $100 \mathrm{~nm}$ and period of $200 \mathrm{~nm}$. 
measurements. Some experimental works have already been performed, but this remains an important topic of study.

\section{Conclusions}

With an increasing capability of structuring materials at a smaller and smaller scale, one can access more and more precise control of light/material interaction. We can expect to organize the propagation of optical waves as well, close to the material in the near field as at any distance of it.

Random surface structures that control statistical properties are developed to shape, at will, the bidirectional reflectance distribution function (BRDF) of surface. Wide-band antireflection structures are rapidly progressing but also deeply etched surfaces like black silicon appear to exhibit remarkably strong absorption and antireflection properties even in the infrared. The inhomogeneities and the structure of thin films can now be partly controlled. This is used to make materials with defined refractive index with, in particular, birefringent properties of high interest for applications.

Photonic crystals have also experienced a very strong development these last years in particular with the metamaterials. New developments are now taking place with nanomagnetic structures. But when the structure can be controlled at a smaller size of a few thousands atoms, a new field is opening with a control of intimate properties of matter.

Quantum confinement, which can be used to tune the properties of electrons, must lead to completely new applications where electrons and photons must be considered together. The concept of permittivity is then revisited.

This concept is also different for metallic nanoparticles embedded in a dielectric material and the association of plasmon resonance and scattering again concerns both electron and photons.

Though not discussed in this paper, the dynamic properties of electrons and the nonlinear optical properties have yet to be jointly considered and a lot of research has yet to be performed in these fields. This should pave the way to more and more complex applications at low cost, well suited to nomadism, for every day life, energy, security, environment, and health.

\section{References}

1. P. Giacomo, "Les couches réfléchissantes multidiélectriques appliquées à l'interférométrie de Fabry-Perot. Étude théorique et expérimentale des couches réelles," Rev. Opt., Theor. Instrum. 35, 317-354 (1956).

2. E. Kröger and E. Kretschmann, "Scattering of light by slightly rough surfaces on thin films including plasma resonance emission," Z. Phys. 237, 1-15 (1970).

3. J. M. Elson and J. M. Bennett, "Relation between the angular dependence of scattering and the statistical properties of optical surfaces," J. Opt. Soc. Am. 69, 31-47 (1979).

4. P. Bousquet, F. Flory, and P. Roche, "Scattering from multilayer thin films: Theory and experiment," J. Opt. Soc. Am. 71(9), 1115-1123 (1981).

5. S. O. Rice, "Reflection of electromagnetic waves from slightly rough surfaces," Commun. Pure Appl. Math. 4, 351-378 (1951).

6. A. A. Maradudin, Light Scattering and Nanoscale Surface Roughness, Springer, New York (2007).

7. A. Beckmann and A. Spizzichino, The Scattering of Electromagnetic Waves from Rough Surfaces, Pergamon press, New York (1963).

8. A. G. Voronovich, Wave Scattering from Rough Surfaces, Springer-Verlag, Berlin (1994).

9. E. Bahar, "Full wave solutions for the depolarization of the scattered radiation fields by rough surfaces of arbitrary slopes," IEEE Trans. Antennas Propag. 29, 443-454 (1981).

10. J. Shen and A. A. Maradudin, "Multiple scattering of waves from random rough surfaces," Phys. Rev. B 22, 4234-4240 (1980). 
11. D. P. Winebrenner and A. Ishimaru, "Investigation of a surface field phase-perturbation technique for scattering from rough surfaces," Radio Sci. 20, 161-170 (1985).

12. V. I. Tatarskii, "The expansion of the solution of the rough surface scattering problem in powers of quasi-slopes," Waves Random Media 3, 127-146 (1993).

13. A. G. Voronovich, "Small-slope approximation in wave scattering by rough surfaces," Sov. Phys. JETP 62, 65-70 (1985).

14. A. G. Voronovich, "Small-slope approximation for electromagnetic wave scattering at a rough interface of two dielectric halfspace," Waves Random Media 4, 337-367 (1994).

15. G. Berginc, "Small-slope approximation method: A further study of vector wave scattering from two-dimensional surfaces and comparison with experimental data," Prog. Electromagnetics Res., PIER 37, 251-287 (2002).

16. G. Berginc and C. Bourrely, "The small slope approximation method applied to a three dimensional slab with rough boundaries," PIER 73, 131-211 (2007).

17. C. Bourlier and G. Berginc, "Multiple scattering in the high-frequency limit with secondorder shadowing function from 2-D anisotropic rough dielectric surfaces: I Theoretical study," Waves in Random Media 14, 229-252 (2004); 14, 253-276 (2004).

18. G. Berginc and C. Bourrely, "Light scattering from 3D nanoscale disordered media," PIERS Online 6(8), 730-734 (2010).

19. A. A. Maradudin, Ed., Structured Surfaces as Optical Metamaterials, Cambridge University, Cambridge, England (2011).

20. P. F. Gray, "Method of forming optical diffusers of simple known statistical properties," Opt. Acta 25(8), 765-775 (1978).

21. E. R. Mendez, M. A. Ponce, V. Ruizcortes, and Z. H. Gu, "Photofabrication of onedimensional rough surfaces for light-scattering experiments," Appl. Opt. 30(28), $4103-$ 4112 (1991).

22. E. R. Mendez, E. E. Garcia-Guerrero, H. M. Escamilla, A. A. Maradudin, T. A. Leskova, and A. V. Shchegrov, "Photofabrication of random achromatic optical diffusers for uniform illumination," Appl. Opt. 40, 1098-1108 (2001).

23. E. R. Mendez, T. A. Leskova, A. A. Maradudin, M. Leyva-Lucero, and J. Munoz-Lopez, "The design of two-dimensional random surfaces with specified scattering properties," J. Opt. A, Pure Appl. Opt. 7, 141-151 (2005).

24. E. E. Garcia-Guerrero, E. R. Mendez, and H. M. Escamilla, "Design and fabrication of random phase diffusers for extending the depth of focus," Opt. Express 15, 910-923 (2007).

25. V. Brissonneau, L. Escoubas, F. Flory, G. Berginc, G. Soriano, G. Maire, and H. Giovannini, "Laser assisted fabrication of random rough surfaces for optoelectronics," to be published in Appl. Surf. Sci. (2011).

26. T.-H. Her, R. J. Finlay, C. Wu, S. Deliwala, and E. Mazur, "Microstructuring of silicon with femtosecond laser pulses," Appl. Phys. Lett. 73, 1673-1675 (1998).

27. L. L. Ma, Y. C. Zhou, N. Jiang, X. Lu, J. Shao, W. Lu, J. Ge, X. M. Ding, and X. Y. Hou, "Wide-band 'black silicon' based on porous silicon,"Appl. Phys. Lett. 88, 171907 (2006).

28. T. P. Chow, P. A. Maciel, and G. M. Fanelli, "Reactive Ion Etching of Silicon in CCl4 and HCl Plasmas," J. Electrochem. Soc. 134, 1281-1286 (1987).

29. T. H. Her, R. J. Finlay, C. Wu, and E. Mazur, "Femtosecond laser-induced for-mation of spikes on silicon," Appl. Phys. A: Mater. Sci. Process. 70(4), 383-385 (2000).

30. C. Wu, C. H. Crouch, L. Zhao, J. E. Carey, R. Younkin, J. A. Levinson, E. Mazur, R. M. Farrell, P. Gothoskar, and A. Karger, "Near-unity below-band gap absorption by microstructured silicon," Appl. Phys. Lett. 78, 1850-1852 (2001).

31. R. Bouffaron, L. Escoubas, J. J. Simon, P. Torchio, F. Flory, G. Berginc, and P. Masclet, "Enhanced antireflecting properties of micro-structured top-flat pyramids," Opt. Express 16(23), 19304-19309 (2008). 
32. L. Escoubas, R. Bouffaron, V. Brissonneau, J. J. Simon, G. Berginc, F. Flory, and P. Torchio, "Sand-castle bi-periodic pattern for spectral and angular broadening of antireflective properties," Opt. Lett. 35(9), 1455-1457 (2010).

33. C. H. Sun, P. Jiang, and B. Jiang, "Broadband moth-eye antireflection coatings on silicon," Appl. Phys. Lett. 92, 061112 (2008).

34. Y. J. Zhang, W. Li, and K. J. Chen, "Application of two-dimensional polystyrene arrays in the fabrication of ordered silicon pillars," J. Alloys Compd. 450(1-2), 512-516 (2008).

35. Y. J. Zhang, X. H. Wang, Y. X. Wang, H. L. Liu, and J. H. Yang, "Ordered nanostructures array fabricated by nanosphere lithography," J. Alloys Compd. 452(2), 473-477 (2008).

36. H. A. Macleod, Thin-Film Optical Filters, 4th ed., Chemical Rubber, Boca Raton (2010).

37. G. Dirks and H. J. Leamy, "Columnar microstructure of optical thin films," Thin Solid Films 47, 219-222 (1997).

38. F. Flory and L. Escoubas, "Optical properties of nanostructured thin films," Prog. Quantum Electron. 28, 89-112 (2004).

39. S. Ogura and H. A. Macleod, "Water sorption phenomena in optical thin films," Thin Solid Films 34, 371-375 (1976).

40. K. Kinosita and M. Nishibori, "Effects of vacuum exposure on stress and spectral shift of high reflective coatings," J. Vac. Sci. Technol. 6, 730-733 (1969).

41. J. C. Maxwell-Garnett, "Colors in metal glasses and in metal films," Philos. Trans. R. Soc. 203, 385-419 (1904).

42. W. L. Bragg and A. B. Pippard, "The form birefringence of macromolecules," Acta Crystallogr. 6, 865-867 (1953).

43. S. Rytov, "Electromagnetic properties of a finely stratified medium," Sov. Phys. JETP 2, 466-474 (1956).

44. H. Jänchen, D. Endelema, N. Kaiser, and F. Flory, "Determination of the refractive indices of highly biaxial anisotropic coatings using guided modes," Pure Appl. Opt. 5(4), 405-415 (1996).

45. F. Flory, D. Endelema, E. Pelletier, and I. Hodgkinson, "Anisotropy in thin films. Modelization and measurement of guided and non guided optical properties. Application to $\mathrm{TiO}_{2}$ films," Appl. Opt. 32(28), 5649-5659 (1993).

46. F. Horowitz and H. A. Macleod, "Form birefringence in thin films," Proc. SPIE 380, 83-87 (1983).

47. K. Robbie, M. J. Brett, and A. Lakhtakia, "Chiral sculptured thin films," Nature 384, 616 (1996).

48. A. Lakhtakia and R. Messier, "Sculptured thin films," Nanometer Structures: Theory, Modeling, and Simulation, A. Lakhtakia, Ed., SPIE Press, Washington, WA (2004).

49. A. Lakhtakia and R. Messier, Sculptured Thin Films: Nanoengineered Morphology and Optics, SPIE Press, Washington, WA (2005).

50. D. Berreman, "Optics in stratified and anisotropic media: $4 \times 4$ matrix formulation," J. Opt. Soc. Am. 62, 502-510 (1972).

51. I. Hodgkinson and H. Wu, Birefringent Thin Films and Polarizing Elements, World Scientific, Singapore (1997).

52. T. Motohiro and Y. Taga, "Thin film retardation plate by oblique deposition," Appl. Opt. 28, 2466-2482 (1989).

53. F. Flory, L. Escoubas, and B. Lazaridés, "Artificial anisotropy and polarizing filters," Appl. Opt. 41(16), 3332-3335 (2002).

54. F. Flory, "Characterization: Guided wave techniques," Thin Films for Optical Systems, F. Flory, ed., Marcel Dekker, New York (1995). 
55. S. Monneret, P. Huguet-Chantôme, and F. Flory, "m-Lines technique: Prism coupling measurement and discussion of accuracy for homogeneous waveguides," J. Opt. A, Pure Appl. Opt. 2(3), 188-195 (2000).

56. J. Massaneda, F. Flory, and E. Pelletier, "Determination of the refractive index of layers in a multilayer stack by guided wave technique," Appl. Opt. 38, 19, 4177-4181 (1999).

57. T. Mazingue, L. Escoubas, L. Spalluto, F. Flory, G. Socol, C. Ristoscu, E. Axente, S. Grigorescu, I. N. Mihailescu, and N. A. Vainos, "Nanostructured $\mathrm{ZnO}$ coatings grown by pulsed laser deposition for optical gas sensing of butane," J. Appl. Phys. 98, 074312 (2005).

58. D. Kossel, "Analogies between thin-film optics and electron band theory of solids," J. Opt. Soc. Am. 568, 1434 (1966).

59. K. Sakoda, Optical Properties of Photonic Crystals, 2nd ed., Springer-Verlag, Berlin (2004).

60. E. M. Purcell, "Spontaneous emission probabilities at radio frequencies," Phys. Rev. 69, 681 (1946).

61. L. Escoubas, E. Drouard, and F. Flory, "Designing waveguide filters with optical thin-film computational tools," Opt. Commun. 197, 309-316 (2001).

62. A. Lakhtakia, Ed., Nanometer Structures: Theory, Modeling, and Simulation, SPIE Press, Washington (2004).

63. P. S. Zory, "The origin of quantum wells and the quantum well laser," in Quantum Well Lasers, C. H. Henry Jr., Ed., Academic, New York (1993), pp. 1-13.

64. H. Schneider and H. Liu, Quantum Well Infrared Photodetectors: Physics and Applications, Springer-Verlag, Berlin (2006).

65. M. Bruchez, M. Moronne, P. Gin, S. Weiss, and A. P. Alvistos, "Semiconductor nanocrystals as fluorescent biological labels," Science 281, 2013-2016 (1998).

66. S. Nizamoglu, G. Zengin, and H. V. Demir, "Color-converting combinations of nanocrystal emitters for warm-white light generation with high color rendering index," Appl. Phys. Lett. 92, 031102 (2008).

67. S. Coe, W. K. Woo, M. Bawendi, and V. Bulovic, "Electroluminescence from single monolayers of nanocrystals in molecular organic devices," Nature 420, 800-803 (2002).

68. W. Shockley and H. J. Queisser, "Detailed balance limit of efficiency of $p / n$ junction solar cells," J. Appl. Phys. 32, 510-519 (1961).

69. P. V. Kamat, "Quantum dot solar cells. Semiconductor nanocrystals as light harvesters," J. Phys. Chem. C 112, 18737-18753 (2008).

70. O. V. Vassiltsova, S. K. Panda, Z. Zhao, M. A. Carpenter, and M. A. Petrukhina, "Ordered fabrication of luminescent multilayered thin films of CdSe quantum dots," Dalton Trans. 9426-9432 (2009).

71. S. A. Studenikin, N. Golego, and M. Cocivera, "Fabrication of green and orange photoluminescent, undoped $\mathrm{ZnO}$ films using spray pyrolysis," J. Appl. Phys. 84(4), 2287-2294 (1998).

72. F. Flory, Y.-J. Chen, C.-C. Lee, L. Escoubas, J.-J. Simon, P. Torchio, J. Le Rouzo, S. Vedraine, H. Derbal-Habak, I. Shupyk, Y. Didane, and J. Ackermann, "Optical properties of dielectric thin films including quantum dots," Appl. Opt. 50(9), C129-C134 (2011).

73. I. Robel, V. Subramanian, M. Kuno, and P. V. Kamat, "Quantum dot solar cells. Harvesting light energy with $\mathrm{CdSe}$ nanocrystals molecularly linked to mesoscopic $\mathrm{TiO}_{2}$ films," $\mathrm{J}$. Am. Chem. Soc. 128, 2385-2393 (2006).

74. A. Kongkanand, K. Tvrdy, K. Takechi, M. Kuno, and P. V. Kamat, "Quantum dot solar cells. Tuning photoresponse through size and shape control of $\mathrm{CdSe}-\mathrm{TiO}_{2}$ architecture," J. Am. Chem. Soc. 130, 4007-4015 (2008).

75. S. Günesa, H. Fritzb, N. S. Neugebauera, S. Sariciftcia, Kumar, and G. D. Scholes, "Hybrid solar cells using PbS nanoparticles," Sol. Energy Mater. Sol. Cells 91(5), 420-423 (2007). 
Flory, Escoubas, and Berginc: Optical properties of nanostructured materials: a review

76. T. Jiu, P. Reiss, S. Guillerez, R. de Bettignies, S. Bailly, and F. Chandezon, "Hybrid solar cells based on blends of CdSe nanorods and poly(3-alkylthiophene) nanofibers," IEEE J Sel Top. Quantum Electron. 16(6), 1619-1626 (2010).

77. R. J. Ellingson, M. C. Beard, J. C. Johnson, P. Yu, O. I. Micic, A. J. Nozik, A. Shabaev, and A. L. Efros, "Highly efficient multiple exciton generation in colloidal $\mathrm{PbSe}$ and $\mathrm{PbS}$ quantum dots," Nano Lett. 5(5), 865-871 (2005).

78. H. A. Lorentz, "Über die Beziehungzwischen der Fortpflanzungsgeschwindigkeit des Lichtes der Körperdichte," Ann. Phys. Lpz. 9, 641-665 (1880).

79. L. Lorenz, "Über die Refractionsconstante," Ann. der Phys. 11, 70-103 (1880).

80. M. Born and E. Wolf, Chapter 2 in Principles of Optics, 7th (expanded) ed., Cambridge University, Cambridge, England (1999).

81. P. Carpena, V. Gasparian, and M. Ortuño, "Number of bound states of a Krönig-Penney finite-periodic Superlattice," Eur. Phys. J. B 8, 635-641 (1999).

82. C. Cohen-Tannoudji, B. Diu, and F. Laloë, Mécanique quantique, Hermann, Paris (1997).

83. H. Li, "Refractive index of interdiffused AlGaAs/GaAs quantum well," J. Appl. Phys. 82(12), 6251-6258 (1997).

84. S. V. Gaponenko, Optical Properties of Semiconductor Nanocrystals, Cambridge University Press, Cambridge, England (1998).

85. E. Kretschmann and H. Raether, "Radiative decay of non-radiative surface plasmons excited by light," Z. Naturforsch. A 23, 2135 (1968).

86. G. Mie, "Beiträge zur optik trüber medien, Speziell Kolloidaler Metallösungen," Ann. Phys. 330, 377-445 (1908).

87. J. A. Polo Jr. and A. Lakhtakia, "Surface electromagnetic waves: A review," Laser Photonics Rev. 5, 234-246 (2011).

88. U. Kreibig and M. Vollmer, Optical Properties of Metal Clusters, Springer-Verlag, Berlin (1995).

89. L. Novotny, "Optical antennas tuned to pitch," Nature 455, 887 (2008).

90. K. Kneipp, Y. Wang, H. Kneipp, L. T. Perelman, I. Itzkan, R. R. Dasari, and M. S. Feld, "Single Molecule Detection Using Surface-Enhanced Raman Scattering (SERS)," Phys. Rev. Lett. 78(9), 1667-1670 (1997).

91. B-S. Yeo, J. Stadler, T. Schmid, R. Zenobi, and W. Zhang, "Tip-enhanced Raman Spectroscopy-Its status, challenges and future directions," Chem. Phys. Lett. 472, 1-13 (2009).

92. W. L. Barnes, A. Dereux, and T. W. Ebbesen, "Surface plasmon subwavelength optics," Nature (London) 424, 824-830 (2003).

93. B. Liedberg, C. Nylander, and I. Lundström, "Surface plasmon resonance for gas detection and biosensing," Sens. Actuators 4, 299-304 (1983).

94. M.-K. Kwon, J.-Y. Kim, B.-H. Kim, I.-K. Park, C.-Y. Cho, C.-C. Byeon, and S.-J. Park, "Surface-plasmon-enhanced light-emitting diodes," Adv. Mater. 20, 1253-1257 (2008).

95. O. Stenzel, A. Stendhal, K. Voigtsberger, and C. Von Borczyskowski, "Enhancement of the photovoltaic conversion efficiency of copper phthalocyanine thin film devices by incorporation of metal clusters," Sol. Energy Mater. Sol. Cells 37, 337-348 (1995).

96. D. Derkacs, S. H. Lim, P. Matheu, W. Mar, and E. T. Yu, "Improved performance of amorphous silicon solar cells via scattering from surface plasmon polaritons in nearby metallic nanoparticles," Appl. Phys. Lett. 89, 093103 (2006).

97. S. Pillai, K. R. Catchpole, T. Trupke, and M. A. Green, "Surface plasmon enhanced silicon solar cells," J. Appl. Phys. 101, 093105 (2007).

98. R. B. Konda, R. Mundle, H. Mustafa, O. Bamiduro, A. K. Pradhan, U. N. Roy, Y. Cui, and A. Burger, "Surface plasmon excitation via Au nanoparticles in n-CdSe/p-Si heterojunction diodes," Appl. Phys. Lett. 91, 191111 (2007). 
99. J. Kim, K. Lee, N. Coates, D. Moses, T. Nguyen, M. Dante, and A. Heeger, "Efficient tandem polymer solar cells fabricated by all-solution processing,." Science 317, 222-225 (2007).

100. D. Duche, P. Torchio, L. Escoubas, F. Monestier, J. J. Simon, F. Flory, and G. Mathian, "Improving light absorption in organic solar cells by plasmonic contribution," Sol. Energy Mater. Sol. Cells 93, 1377-1382 (2009).

101. S. Vedraine, P. Torchio, D. Duché, F. Flory, J.-J. Simon, J. Le-Rouzo, and L. Escoubas, "Intrinsic absorption of plasmonic structures for organic solar cells," in Sol. Energy Mater. Sol. Cells 95, S57-S64 (2011).

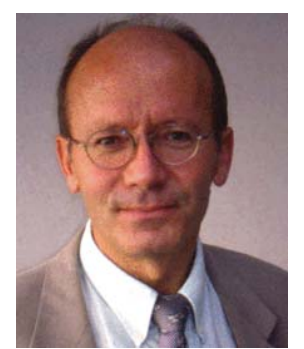

Francois Flory graduated from Ecole Centrale Marseille in 1975, then received a PhD in optics in 1978 and a "Thése d'Etat" in 1987. He is currently a professor "classe exceptionnelle" at Ecole Centrale Marseille. He was deputy director of this institution for 6 years and co-founded the optical cluster POPsud. He performs his research at the Institute for Materials, Microelectronics and Nanosciences, Mareseille, France. His research interests are optical coatings and optical properties of nanostructured materials for different application fields such as solar cells, sensors, and light detectors. He is editor and co-author of the book Thin Films for Optical Systems (Marcel Dekker, 1995) and author of more than 200 publications, book chapters, patents, and conferences. He was the chairman of the conference Education and Training in Optics and Photonics (2005). He has been member of scientific committees of several international conferences and is a reviewer for several international scientific journals.

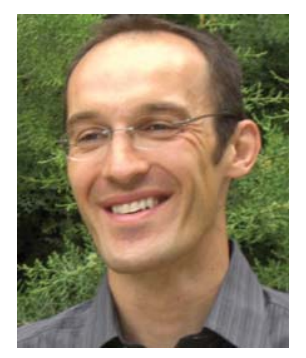

Ludovic Escoubas graduated from Centrale Marseille and received a $\mathrm{PhD}$ in optics in 1997. He is now a professor at Paul Cezanne University, Marseille, France and leader of the Optoelectronics Components and Photovoltaics (OPTO-PV) Team of IM2NP (CNRS Laboratory). His current research interests are micro- and nano-optical components and solar cells. He has authored more than 150 papers and communications, and he holds 6 patents.

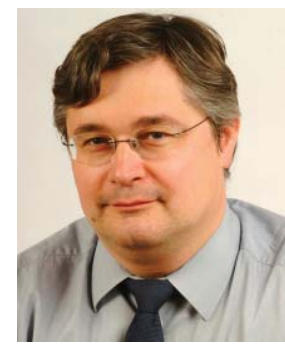

Gérard Berginc received a Dipl. Engineer-Physicist degree from Ecole Centrale de Marseille and his doctorate in theoretical physics. He is currently a chief scientist and head of Advanced Research and Technology at Thales Optronique S.A. His research activities include high-frequency asymptotics, random volume and rough surface scattering, coherent effects in random media, and localization phenomena. He has authored or co-authored over 150 publications in books, journals and conferences, and is a Fellow Member of the Electromagnetics Academy. He holds more than 60 patents and was listed in Who's Who in Electromagnetism and Who's

Who in the World. 